\title{
Caracterización de estudiantes de la carrera de enfermería sobre consumo de drogas lícitas e ilícitas
}

\author{
Viriam Leiva Diaz $^{1}$ \\ Gabriela Pereira Vasters ${ }^{2}$ \\ Moacyr Lobo da Costa Jr. ${ }^{3}$
}

\begin{abstract}
Se trata de un estudio cuantitativo, descriptivo y transversal que buscó caracterizar el uso de drogas entre estudiantes del segundo año de la carrera de Licenciatura en Enfermería. Se aplicó un cuestionario autoadministrado a 119 estudiantes, el que se dividió en información: general, sociodemográfica, económica, personal, familiar, sociocultural y, sobre el consumo de drogas. El estudio evidenció que los estudiantes poseen factores protectores para la prevención del consumo o el avance del uso problemático de drogas; pero, por otro lado poseen también factores de riesgo importantes que deben ser tomados en cuenta en programas de prevención, por ejemplo: estilos de vida no saludables, consumo de drogas de personas cercanas a ellos (familiares, amigos o compañeros). A pesar de que el alcohol y tabaco ocupan el primer lugar de consumo, tanto en el entorno inmediato del grupo de estudiantes seleccionados como en el ámbito nacional, ellos no lo consideran un problema tan grande si comparado con las drogas ilícitas.

Descriptores: Trastornos Relacionados con Sustancias; Drogas Ilícitas; Estudiantes de Enfermería.
\end{abstract}

\footnotetext{
${ }^{1}$ Psicóloga y Enfermera, Maestría en Psicopedagogía, Profesor Asociado, Escuela de Enfermería, Universidad de Costa Rica, Costa Rica. E-mail viriaml@yahoo.es.

${ }^{2}$ Estudiante de Doctorado, Escola de Enfermagem de Ribeirão Preto, Universidade de São Paulo, Centro Colaborador de la OMS para el Desarrollo de la Investigación en Enfermería, Brasil. E-mail: gabi_vastesr@yahoo.com.br.

${ }^{3}$ Estadístico, Doctor em Salud Publica, Profesor Asociado, Escola de Enfermagem de Ribeirão Preto, Universidade de São Paulo, Centro Colaborador de la OMS para el Desarrollo de la Investigación en Enfermería, SP, Brasil. E-mail: mlobojr@eerp.usp.br.
}

Correspondencia:

Moacyr Lobo da Costa Jr.

Universidade de São Paulo. Escola de Enfermagem de Ribeirão Preto

Av. Bandeirantes, 3900

Bairro Monte Alegre

CEP: 14040-902 Ribeirão Preto, SP, Brasil

E-mail: mlobojr@eerp.usp.br 


\title{
Caracterização de estudantes do curso de enfermagem sobre o consumo de drogas lícitas e ilícitas
}

Trata-se de estudo quantitativo, descritivo e transversal que buscou caracterizar o uso de drogas entre estudantes de segundo ano de Licenciatura de Enfermagem. Aplicouse questionário autoadministrado a 119 estudantes, contendo informações gerais, sociodemográficas, econômicas, pessoais, familiares, socioculturais e sobre o consumo de drogas. $O$ estudo evidenciou que os estudantes possuem fatores protetores para a prevenção do consumo ou avanço ao uso problemático de drogas, embora possuam também fatores de risco importantes como estilos de vida não saudáveis e consumo de drogas de pessoas próximas (familiares, amigos e companheiros). Além disso, ficou evidente que o perfil de consumo de drogas tanto lícitas como ilícitas é similar ao que se observa no cenário nacional e internacional. Importante destacar que, apesar do consumo de álcool e tabaco ocupar o primeiro lugar, tanto para os estudantes selecionados como em nível nacional, eles não o consideram problema tão grande comparado com drogas ilícitas.

Descritores: Transtornos Relacionados ao uso de Substâncias; Drogas Ilícitas; Estudantes de Enfermagem.

\section{Characterization of the Students of a Nursing Course Regarding the Consumption of Licit and Illicit Drugs}

\begin{abstract}
The aim of this quantitative, descriptive, transversal study was to characterize drug use among students in the second year of the Nursing Degree course. The sample of 119 students answered a questionnaire divided into: general information, sociodemographic, economic, personal, familiar, sociocultural and drug usage. The study showed that students possess factors that protect them from drug usage and the acquisition of drug habits; but that they also have risk factors which must be taken into consideration in prevention programs, such as unhealthy life styles or drug abusage in people close to them (family, friends or classmates). We found that the profile of drugs used, containing both legal and illegal drugs, was similar to those in the national and international context. It is important to emphasize that even though the consumption of alcohol and tobacco occupy the highest places, in the student environment as well as nationally, these are not considered as problematic as the illegal drugs.
\end{abstract}

Descriptors: Substance-Related Disorders; Street Drugs; Students, Nursing.

\section{Introducción}

Tanto a nivel mundial como nacional el uso y abuso de las drogas, lícitas o ilícitas, son una preocupación creciente, en el ámbito político, social, económico y de la salud, no solo por las implicaciones que causa en la persona - modificaciones químicas o físicas de su organismo, mediante una acción directa en el sistema nervioso central lo que altera su estructura y funcionamiento(1) - como también en el ámbito familiar, social y mundial. Para esto basta con mencionar el Informe de la Oficina contra la Droga y el Crimen de la Organización de las Naciones Unidas del año 2004, en el cual los consumidores de sustancias ilegales llegan a 185 millones de personas; en el año 2005 este porcentaje, en la población mundial, se incrementó en un $5 \%{ }^{(2)}$.

El consumo de drogas lícitas e ilícitas ocasiona enfermedades y trastornos en los usuarios, que llegan a sufrir incapacidades o ser la causa de su muerte, también producen efectos físicos en un período corto de tiempo (adicción) en una población en edad productiva, 
lo que ocasiona costos en el ámbito económico, como el pago de incapacidades temporales o permanentes; así como efectos sociales derivados del consumo, como el daño en las relaciones interpersonales, la pérdida del trabajo, la desintegración familiar, entre otros.

En Costa Rica según datos del Informe Consolidado sobre los costos del problema de las drogas ilegales en Costa Rica entre los años 2000-2003(2), estos impactaron principalmente en los costos gubernamentales directos y en particular los referentes a la reducción de la oferta. A estos le siguieron los costos por pérdida de productividad, que presentan un pequeño aumento pero continuo hasta 2002, y luego un descenso en 2003. Los costos en salud no presentan grandes variaciones $y$ tienen una evolución muy similar a la de los costos por pérdida de productividad.

Algunos de los factores de riesgo y protectores en el uso y consumo problemático de las $\operatorname{drogas}^{(3)}$ que se pueden mencionar son: autoestima(4-5); aspectos psicológicos como ansiedad, depresión y estrés que están asociados al consumo de drogas (ya que constituyen un medio rápido de aliviar las sensaciones corporales asociados al malestar emocional, por sus efectos directos sobre el sistema nervioso central(6) ${ }^{(6)}$; inhabilidad social e insatisfacción en las relaciones interpersonales (dificultad en las personas para defender sus opiniones frente a los diferentes grupos, lo cual dificulta que pueda rechazar la oferta de drogas; así como, la creencia de que el consumo de drogas lo puede ayudar a ser aceptado por el grupo de iguales $\left.{ }^{(3)}\right)$; habilidades emocionales (disminuyen la probabilidad de consumo, ya que a través del autoconocimiento, la identificación, expresión y manejo de sentimientos, redunda en el mejor manejo del estrés y la ansiedad de una manera positiva( $\left.{ }^{(7)}\right)$; los prejuicios y la valoración de la letalidad de las drogas (la baja percepción de riesgo, de sus efectos y consecuencias lleva al consumo de las mismas $\left.{ }^{(8)}\right)$; y, la espiritualidad como un factor protector que previene el consumo inicial y facilita la abstinencia en los procesos de recuperación de la adicción ${ }^{(9)}$. El maltrato en todas sus formas y la disfunción familiar, han sido reconocidos como un factor de predicción del consumo de drogas. El primero se ha asociado a la inducción temprana y el segundo a los primeros consumos en adolescentes ${ }^{(8)}$.

\section{Objetivos}

El objetivo de este estudio fue caracterizar el consumo de drogas, lícitas o ilícitas, entre estudiantes de enfermería en una universidad de Costa Rica.

\section{Materiales y Métodos}

Este artículo es una extensión del proyecto de investigación No 200, presentado y aprobado por la Vicerrectoría de Investigación, titulado "factores sociales, culturales, personales, familiares y económicos asociados al fenómeno de las drogas en estudiantes de la carrera de Licenciatura en Enfermería: un estudio desde la perspectiva de la Salud Internacional", de la Escuela de Enfermería de la Universidad de Costa Rica.

Es un estudio cuantitativo, descriptivo y transversal. Para el presente artículo la población estuvo constituida por 119 estudiantes que cursaban el II año de la carrera en Enfermería.

Para la recolección de la información se construyó un cuestionario de factores sociales culturales, personales, familiares y económicos, asociados al consumo de drogas, y por tanto, el instrumento se dividió en las siguientes partes: información general, sociodemográfica, económica, personal, familiar y sociocultural y, consumo de drogas. Antes de aplicar el cuestionario, los sujetos fueron informados sobre su participación en el estudio, sobre los fines del mismo, así como la confidencialidad de sus respuestas, todo esto se concretizó con la firma del Consentimiento Informado. La recolección de información se llevó a cabo durante dos meses de manera grupal y autoaplicada. Para el análisis de resultados se utilizó el Statistical Package for Social Sciences [SPSS] versión 13.0 para Windows.

\section{Resultados}

Se encontró que la carrera de Enfermería es eminentemente femenina, $84,87 \%$ de los encuestados son del sexo femenino. Además es importante mencionar que el $96,6 \%$ de ellos son menores de 25 años de edad, destacándose entre estos el 65.5\% que tiene 20 o menos años de edad. Además con respecto al estado civil un $97,5 \%$ eran solteros y solteras.

Un factor protector que se analiza dentro del consumo de drogas es el aspecto familiar, en el cual se distingue la estabilidad familiar como fortaleza para el no consumo de drogas. Con respecto a esto entre los estudiantes (Tabla 1), fue verificado que persiste el perfil de familia nuclear en un grupo importante del estudiantado, tanto en tiempo lectivo como en el no lectivo viven con sus padres y hermanos. Cabe mencionar que las variaciones en cuanto al rubro de compañeros y amigos en tiempo no lectivo disminuyen ya que, en esta época las estudiantes que viven lejos regresan con sus familias de origen. 
Tabla 1 - Distribución de los estudiantes según persona con quien vive en tiempo lectivo y no lectivo, 2007

\begin{tabular}{lccccc}
\hline \multirow{2}{*}{ Persona } & \multicolumn{2}{c}{ En tiempo lectivo } & & \multicolumn{2}{c}{ En tiempo no lectivo } \\
\cline { 2 - 3 } \cline { 5 - 6 } & $\mathbf{N}$ & $\%$ & & $\mathbf{N}$ & $\%$ \\
\hline Solo & 05 & 4,2 & & 01 & 0,8 \\
Papá & 41 & 34,5 & & 77 & 64,7 \\
Mamá & 54 & 45,5 & & 99 & 83,2 \\
Hermanos & 57 & 47,9 & & 91 & 76,5 \\
Hijos & 02 & 1,7 & & 02 & 1,7 \\
Pareja & 06 & 5,0 & & 04 & 3,4 \\
Familiares & 16 & 13,4 & & 17 & 14,3 \\
Compañeros & 32 & 26,9 & & 02 & 1,7 \\
Amigos & 22 & 18,5 & & 03 & 2,5 \\
\hline
\end{tabular}

En cuanto a la escolaridad de los padres de los encuestados, un $33.6 \%$ posee estudios universitarios, un $22,7 \%$ tenía secundaria completa y un $16.8 \%$ primaria completa.

La fuente de ingreso económico familiar del grupo encuestado provino principalmente del salario (74,8\%), y la persona que lo aportaba era el padre, en primer lugar con un $72,3 \%$, seguido de la madre en un $48,7 \%$. Cabe mencionar que ambas opciones no fueron excluyentes, es decir que, puede ser el padre, la madre o ambos. El resto del porcentaje se distribuye entre hermanos y familiares. El ingreso mensual familiar, en un $50,4 \%$, se encontraba entre $100 \mathrm{mil}$ a $300 \mathrm{mil}$ colones (esto es entre $\$ 200$ y $\$ 600)$, el resto está distribuido entre menos de 100 mil colones $(16,8 \%)$ a más de 300 mil colones $(28,6 \%)$.

De los 119 encuestados, un 89,1\% no trabajaba; un $10,9 \%$ sí trabajaba, de estos un 5\% realizaba actividades no registradas, es decir, que no estaba cubierto por las garantías otorgadas por ley, por ejemplo el pago de salarios mínimos, el pago de seguro social (el cual cubre enfermedad, maternidad, invalidez y muerte). De los jóvenes que trabajaban un $66,6 \%$ lo hacía en tiempo parcial, es decir menos de 6 horas diarias.

Todo lo anterior lleva a los estudiantes a solicitar becas, a las que pueden aspirar cualquier estudiante de bajos recursos económicos dentro de la Universidad de Costa Rica. Así, tenemos que del total, el $89,1 \%$ disfrutaba de algún tipo de beca, de estos un $41,2 \%$ poseían beca $11^{*}$, ésta incluye la exoneración del pago de matrícula, préstamo de libros semestral o anual, pago de la alimentación y en algunos casos pago de una mensualidad. Un 10,1\% tiene beca categoría 10 , en esta categoría, el estudiante recibe un beneficio igual a la beca categoría 11, con excepción de pago de una mensualidad. $Y$, un $12,1 \%$ recibe algún otro tipo que escalona porcentualmente el pago de la matrícula, por último cabe mencionar que tan sólo $1 \%$ no tiene beca. De los estudiantes que reciben algún tipo de beca el 55,5\% considera que ella es insuficiente para cubrir los gastos relacionados a los estudios y a su manutención.

Otro aspecto que se consideró necesario explorar en términos de intereses y expectativas personales, es la carrera de Enfermería propiamente dicha; para empezar, es importante mencionar que existe una motivación intensa con respecto a la finalización de los estudios y mejora económica futura, esto estaba asociado con su grado de satisfacción, el 69,7\% refiere estar satisfecho con su elección profesional y en regular satisfacción un $21,8 \%$.

Cabe mencionar que el $52,1 \%$ pensó en algún momento en abandonar la carrera, sin embargo conforme avanza en la carrera disminuye el deseo de abandonarla.

Los estudiantes opinan que su desempeño en la carrera es muy bueno con un $67,2 \%$, deficiente y regular $31,1 \%$. Estos datos se confirman, ya que en el último semestre previo a la encuesta, el 63,9\% aprobó todas las materias, un 18,5\% presentó examen de ampliación y aprobó todas las materias matriculadas.

Desde el punto de vista de estilo de vida saludable, es importante conocer a que dedican el tiempo no lectivo, cuales son los sitios más frecuentados.

Los estudiantes en tiempo no lectivo dentro del campus, visitan con más frecuencia la biblioteca $(67,3 \%)$, la cantina $(63 \%)$ y los centros de computación $(61,5 \%)$; entre los menos visitados por los estudiantes se encuentran el CATE (94,9\%) - que es un centro de simulaciones, donde los estudiantes pueden realizar procedimientos de enfermería, la Asociación de Estudiantes $(92,4 \%)$ y las áreas deportivas $(89,1 \%)$.

Tabla 2 - Distribución de los estudiantes según persona a la que acuden en caso de un problema, 2007

\begin{tabular}{lcc}
\hline \multicolumn{1}{c}{ Persona } & Número & Frecuencia $\%$ \\
\hline Madre & 96 & 80,7 \\
Padre & 44 & 37,6 \\
Hermanos & 46 & 38,7 \\
Pareja & 47 & 39,5 \\
Amigos & 81 & 68,1 \\
Familiares & 24 & 20,2 \\
Profesor & 9 & 7,6 \\
Otros & 2 & 1,7 \\
\hline
\end{tabular}


Se evidencia que los jóvenes acuden en primera instancia a la madre dentro de su grupo familiar y fuera de este a los amigos y a la pareja, esto es especialmente importante ya que la consejería que brinda el grupo de iguales puede tener impacto positivo o negativo en las acciones de los jóvenes que solicitan apoyo (Tabla 2).

Con respecto a las relaciones interpersonales que reflejan la autopercepción que se tiene dentro de un grupo determinado, los encuestados tienen está área claramente definida, es así como, el 92,9\% percibe que su opinión y sentimientos dentro del grupo de iguales o de amigos son aceptados y pueden manifestarlos aun cuando el grupo los acepte o no.

Otro aspecto importante que se destaca como posible factor protector en el consumo de drogas es la religión. El 73,9\% de los encuestados profesan la religión católica, esto coincide con el perfil religioso de los costarricenses. El 21,7\% profesa otra religión y un $9,2 \%$ refiere no profesar ninguna religión.

El 62.2\%, del grupo de estudiantes seleccionados, manifiesta que han consumido drogas lícitas e ilícitas alguna vez en su vida. Aquellos que no han probado drogas, dentro de las causas expresadas para el no consumo se encuentra en primer lugar que conocían los efectos secundarios de las drogas $(33,6 \%)$, luego que no les llamaba la atención $(33,6 \%)$, y otros afirmaron que no lo necesitaban $(30,3 \%)$.

Tabla 3 - Distribución de parentesco de la persona que ofreció drogas por primera vez, según los estudiantes

\begin{tabular}{|c|c|c|c|c|c|c|}
\hline \multirow[t]{2}{*}{$\begin{array}{l}\text { Relación de } \\
\text { parentesco }\end{array}$} & \multicolumn{2}{|c|}{$\begin{array}{l}\text { Total de los } \\
\text { estudiantes }\end{array}$} & \multicolumn{2}{|c|}{$\begin{array}{c}\text { Estudiantes que } \\
\text { consumieron }\end{array}$} & \multicolumn{2}{|c|}{$\begin{array}{l}\text { Estudiantes } \\
\text { que consumen } \\
\text { actualmente }\end{array}$} \\
\hline & $\mathbf{n}$ & $\%$ & $\mathbf{n}$ & $\%$ & $\mathbf{n}$ & $\%$ \\
\hline Amigos & 32 & $26,9 \%$ & 70 & $58,8 \%$ & 65 & $54,7 \%$ \\
\hline Compañeros & 15 & $12,6 \%$ & 37 & $31,1 \%$ & 41 & $34,1 \%$ \\
\hline Familiares & 09 & $7,6 \%$ & 24 & $19,3 \%$ & 06 & $4,7 \%$ \\
\hline Pareja & 04 & $3,4 \%$ & 06 & $5,0 \%$ & 02 & $1,6 \%$ \\
\hline
\end{tabular}

Es importante mencionar que los datos de la Tabla 3 se obtuvieron de ítems de respuestas múltiples, es decir, es posible que algunos encuestados señalaron una o más opciones.

Al $41,3 \%$ de los que consumieron drogas tanto lícitas como ilícitas, se les ofreció por primera vez la droga antes de los 15 años de edad; vale la pena mencionar que hubo estudiantes que refirieron que se les ofreció antes de los 15 años de edad.

Se encontró que el $80,7 \%$ de los estudiantes no tenía problemas en cuanto al consumo de alcohol ya que el rubro que presentó el mayor porcentaje de respuesta afirmativa $(32,2 \%)$ fue el que preguntaba si alguna vez sintieron que deberían disminuir la cantidad de bebida o parar de beber; para los otros tres ítems diagnósticos el porcentaje positivo varió entre 1 y $2 \%$. Se encontró dos estudiantes que presentan uso problemático de alcohol

A esta altura podemos destacar que se encontró diferencia entre el consumo de drogas lícitas con respecto a las actividades antes mencionadas; así el consumo de alcohol es mayor en las actividades familiares (52,9\%) con respecto a las religiosas $(45,4 \%)$; el tabaco tiene una relación inversa al alcohol es decir, es mayor en las actividades religiosas $(62,2 \%)$ que en las actividades familiares $(45,4 \%)$. Sin embargo esto no coincide con la frecuencia con que se ofrece drogas en dichas actividades, ya que el porcentaje mayor se ubica en poco frecuente, sin embargo en el rango de frecuente y poco frecuente el consumo mayor sigue siendo de las bebidas alcohólicas y del tabaco Es importante destacar que algunos de los encuestados refirieron que se ofrecían drogas ilícitas, frecuentemente, en las actividades antes mencionadas $(5,0 \%)$.

Tabla 4 - Distribución de motivo de consumo de drogas en el ámbito social, 2007

\begin{tabular}{lcc}
\hline \multicolumn{1}{c}{ Motivo } & n & $\%$ \\
\hline Pertenecer al grupo & 27 & 22,7 \\
Relacionarse con otros & 38 & 31,9 \\
Diversión o placer & 76 & 63,9 \\
Aumentar el desempeño sexual & 07 & 5,9 \\
Ser respetado & 15 & 12,6 \\
Ser más apreciado & 14 & 11,8 \\
Acompañar a la pareja o amigos & 50 & 42,0 \\
\hline
\end{tabular}

Con respecto a Tabla 4, es importante indicar que los ítems no eran mutuamente excluyentes y que los encuestados podían marcar más de una opción; estos refirieron que, entre las razones por las cuales se consume drogas en el ámbito social se encuentra en primer lugar el consumo de drogas por diversión o placer $(63,9 \%)$, en segundo lugar para acompañar a la pareja o amigos $(42,0 \%)$ y en tercer lugar para relacionarse con otras personas $(31,9 \%)$. Un dato importante que refleja la problemática del consumo de drogas y también su efecto en el ámbito social, es la relación del consumo de drogas y accidentes de transito, este indicador fue muy bajo entre los encuestado ya que la asociación del consumo de drogas licitas e ilícitas con los accidentes de tránsito es muy baja varía de 0,8\% a 1,7\%.

Con respecto a la exposición a situaciones de riesgo en su estilo de vida, los estudiantes refieren tener alta 
probabilidad de presentar estilos poco saludables como son el consumo de alimentos con alto contenido de grasa $(29,4 \%)$, vida sedentaria $(20,2 \%)$, consumo de alcohol $(22,7 \%)$ y relaciones sexuales sin protección $(13,4 \%)$. En la escala de mayor probabilidad aparecen los rubros anteriores con excepción de relaciones sexuales que es sustituida por la inhalación de cualquier solvente (27\%).

Asociado siempre al ámbito familiar se indagó sobre el tipo de droga frecuentemente consumida y su relación con el parentesco de la persona que la consume y el estudiantado encuestado. Con respecto al consumo de alcohol, se encontró que el primer lugar lo ocupan los tíos $(23,5 \%)$, el segundo lugar el padre (16\%) y el tercer lugar los hermanos $(8,4 \%)$. En relación con el consumo de tabaco, el primer lugar lo ocupan las madres (25\%), en segundo lugar los tíos $(21,8 \%)$ y los hermanos ocupan el tercer lugar (9,2\%). Llama la atención el alto consumo de tabaco por parte de las madres, ya que denota cambios culturales en lo que se refiere a que el consumo de tabaco y alcohol estuvo por muchos años predominantemente realizado por los hombres.

Además de lo anterior, de los encuestados el $64,2 \%$ (77) refiere tener por lo menos un familiar que consume drogas. De estos, el $44 \%$ mencionó que entre las causas de inicio de consumo de drogas por parte del familiar fueron los problemas encontrados con familiares, con amigos o en el trabajo. El 18,2\% refirió que dicho familiar estuvo envuelto en un accidente de tránsito en el cual se encontró consumo de drogas.

Dentro de las situaciones que influyeron sobre los familiares para el consumo problemático de drogas, se encuentran en primer lugar los problemas familiares $(53,2 \%)$, con la pareja $(28,6 \%)$, con los hijos $(26 \%)$, laborales $(18,2 \%)$ y por último con los estudios $(10,4 \%)$. Todos estos datos apuntan a la problemática que se vive alrededor del consumo de drogas que afecta al individuo y también al grupo familiar nuclear y extenso. Lo más preocupante es que tan solo el $13 \%$ de ellos han buscado algún tipo de ayuda.

Como se puedo observar en los párrafos previos, dentro de las personas que ofrecieron algún tipo de droga a los jóvenes encuestadas, se encuentran los amigos o compañeros, por lo que es importante conocer la situación de consumo de drogas dentro de estos mismos grupos, encontrándose que dentro de los amigos más cercanos, estos consumen en primer lugar alcohol $(88,2 \%)$, seguido por el tabaco $(84,9 \%)$, en tercer lugar la marihuana $(28,6 \%)$ y en cuarto lugar se encuentran la acetona, heroína y solventes con un $17,6 \%$ respectivamente.

\section{Discusión}

La presente investigación encontró que la droga más consumida por estudiantes universitarios es el alcohol, seguida por el tabaco; lo que corresponde al mismo perfil encontrado en el ámbito nacional e internacional(11); también existe un bajo consumo de drogas ilícitas, dentro de estas, las más consumidas son la marihuana y la cocaína.

La edad de inicio (15 años) de consumo de drogas en los estudiantes encuestados fue similar al promedio nacional $(17,13$ años)(11).

En cuanto al género llama la atención que dentro del consumo de drogas en el ámbito familiar hay diferencias, ya que se encontró que el consumo de tabaco es mayoritariamente femenino.

Según los factores de riesgo y protección establecida(12), se detectó que el grupo de estudiantes encuestados poseen más factores protectores que de riesgo, dentro de estos podemos mencionar la estabilidad de la estructura y dinámica familiar, que satisface los procesos y funciones de la familia, la autoestima y sentido de pertenencia familiar social, así como la posibilidad de proyectos de vida viables en congruencia con la escala de valores y morales apropiados.

El comportamiento de consumo de drogas lícitas e ilícitas, que incluyen el alcohol y el tabaco, fue consecuente con los hallazgos encontrados(13-14) en términos de que el consumo de la mayor por parte de los estudiantes encuestados, de sus amigos y de sus familiares, se encuentra predominantemente asociado al uso del alcohol y el tabaco.

Los resultados revelan además que el consumo de drogas tanto lícitas como ilícitas tiene un importante componente social; las expectativas positivas dadas al consumo primordialmente del alcohol por su poder de desinhibidor social, por su capacidad de enfrentamiento de situaciones difíciles tanto en el ámbito social como familiar $y$, por la mejoría de las relaciones vinculares a nivel interpersonal(10), podría convertirse en un factor causal del fuerte mantenimiento de la conducta de beber o del desarrollo abusivo del alcohol(15) en los jóvenes que dicen consumirlo, ya que subestiman la cantidad de alcohol consumido por sí mismos y por sus compañeros(16).

Aun cuando no se puede, en este momento, establecer como un valor predictivo la relación entre tener personas cercanas consumidoras o no, este aspecto si ha sido estudiado y en esta investigación se considera relevante rescatar que los estudiantes 
poseen familiares que presentan problemas en el uso de drogas, así como un porcentaje importante de amigos que también ingieren frecuentemente algún tipo de droga; uno de los mejores factores de predicción de la utilización de drogas es el hecho de tener amigos o familiares que ingieran, por otro lado se convierte en un factor protector, en aquellos jóvenes que reciben desaprobación por parte de su grupo de iguales en lo referente al uso de mismas ${ }^{(13)}$.

\section{Conclusiones}

Por lo tanto, se concluye que a pesar de que el grupo estudiado presenta diferentes factores de protección para el consumo de drogas (la estructura de la estabilidad relativa y dinámica familiar, el cumplimiento de los procesos y funciones de la familia, el sentido de pertenencia a la familia, la autoestima social, la posibilidad de contar con proyectos de vida viables), las drogas lícitas como el alcohol y el tabaco, especialmente, todavía están presentes en contextos sociales, donde son usadas con el objetivo de hacer frente a situaciones de conflicto o para facilitar las interacciones sociales. Entre el estudiantado hay una tolerancia y aceptación mayor del consumo de alcohol y tabaco - en actividades familiares, sociales, religiosas - que el consumo de drogas ilícitas, esto está asociado a la concepción del término licito (legal) o ilícito (ilegal).
En este sentido, las intervenciones necesarias deberían trabajar en nuevas formas que permitan a los estudiantes enfrentar los problemas y establecer lazos emocionales, con el fin de prevenir un uso de riesgo de drogas. Por otra parte, también hay que pensar en las discusiones y propuestas en favor de un estilo de vida más saludable, ya que el sedentarismo en edades tempranas, en combinación con un consumo excesivo de tabaco, alcohol u otras drogas, predisponen a los estudiantes a las complicaciones tempranas de salud física.

\section{Agradecimientos}

Agradecemos a la Comisión Interamericana para el Control del Abuso de Drogas/CICAD de la Secretaria de Seguridad Multidimensional/SSM de la Organización de los Estados Americanos/OEA, la Secretaria Nacional de Políticas sobre Drogas/SENAD do Gabinete de Seguridad Institucional/Brasil, la Escuela de Enfermería de Ribeirao Preto de la Universidad de Sao Paulo y Centro Colaborador de la Organización Mundial de la Salud para el Desarrollo de la Investigación en Enfermería, la población representada en los estudios de investigación, bien como a las autoridades de las universidades representadas por los participantes del Programa En-Line de Especialización en Investigación sobre el Fenómeno de las Drogas PREINVEST, periodos 2005, 2006, 2007 y 2008.

\section{Referencias}

1. World Health Organization. The World Health Report. 2002. [acceso en: 25 noviembre 2007]. Disponible en: http://www. who.int/whr/2002/media_centre/en/index.html.

2. Oficina de las Naciones Unidas contra la Droga y el Delito (ONUDD). Informe Mundial sobre las drogas: 2004. [acceso en: 10 abril 2010]. Disponible en: http://www.unodc.org/pdf/ WDR_2004/wdr2004_vol1_spanish.pdf

2. Pérez A, Valencia J, Rodríguez C. Informe Consolidado sobre los costos del problema de las drogas ilegales en Costa Rica: 2000-2003. 2004. [acceso en: 12 abril 2008]. Disponible en: http://www.cicad.oas.org/oid/MainPage/Costs/Informe $\% 20$ Final\%20Costa\%20Rica.pdf

3. Carballo JL, García O, Secades R, Fernández JR, García E, Erraste JM, et al. Construcción y validación de un cuestionario de factores de riesgo interpersonales para el consumo de drogas en la adolescencia. Psicothema. 2004;16:674-9.

4. Gutierrez-Baró E, Aneiros-Ribas R. Toxicomanías y adolescencia: Realidades y consecuencias. La Habana: Clínica del Adolescente; 1999. 88 p.

5. Malcolm B. Evaluating the Effects of Self-Esteem on Substance Abuse among Homeless Men. J Alcohol Drug Educ. $2004 ; 48: 39-61$.
6. Wu L, Sterling S, Weisner C. Family environment factors and substance abuse severity in an HMO adolescent population. Clin Pediatr. 2004; 43:323-33

7. Griffin K, Scheier L, Botvin G, Díaz T. Protective role of personal competence skills in adolescent substance use: Psychological well-being as a mediating factor. Addict Behav. 2001; 15:194203.

8. Diez JP, Peirats EB. El consumo abusivo de alcohol en la adolescencia: un modelo explicativo desde la psicología social. 1999. [acceso en: 10 diciembre 2009]. Disponible en: http:// www.mir.es/pnd/publica/html/otras.htm.

9. Botella JN. Factores de riesgo y protección de carácter social relacionados con el consumo de drogas. 2000. [acceso en: 10 diciembre 2009]. Disponible en: http://www.onudd.org.pe/ consorciomunicipal/publicaciones/1k-factores.pdf

10. Oliveira EB, Furegato ARF. O trabalho do acadêmico de enfermagem como fator de risco para o consumo de álcool e outras drogas. Rev. Latino-Am. Enfermagem. 2008; 16(n. esp):565-71.

11. Arkowitz H, Hinton R, Perl J, Himadi W. Treatment strategies for dating anxiety in college men based on real-life practice. Couns Psychol. 1978; 7:41-6. 
12. Orozco JB, Montero FU. Consumo de drogas en Costa Rica: resultados de la encuesta nacional 2000-2001. San José: Instituto sobre Alcoholismo y Farmacodependencia (IAFA); 2003.

13. Cáseres D, Salazar I, Varela M, Tobar J. Consumo de drogas en jóvenes universitarios y su relación de riesgo y protección con los factores psicosociales. Universitas Psicol. 2006; 5(3):521-34.

14. Zárate M, Zavaleta A, Danjoy D, Chanamé E, Prochazka R, Salas $M$, et al. Prácticas de consumo de tabaco y otras drogas en estudiantes de ciencias de la salud de una universidad privada de Lima, Perú. Invest Educ Enferm. 2006; 24(2):72-81.
15. Mora-Ríos J, Natera G, Villatoro J, Villalvazo R. Validez factorial del cuestionario de expectativas hacia el alcohol (AEQ) en estudiantes universitarios. Psicol Conductual. 2000; 8: 319-28.

16. Bustamante IV, Carvalho AMP, Oliveira EB, Oliveira Júnior HP, Santos Figueroa SD, Montoya Vásquez EM, et al . University students' perceived norms of peers and drug use: a multicentric study in five Latin American countries. Rev. Latino-Am. Enfermagem. [periódico na Internet]. 2009 [acceso en: 18 abril 2010]; 17(spe):838-43. Disponível em: http:// www.scielo.br/scielo.php?script=sci_arttext\&pid = S0104$11692009000700013 \&$ lng $=$ pt. 